\title{
Prevalência de sobrepeso e obesidade em crianças e adolescentes das regiões Sudeste e Nordeste
}

\author{
Overweight and obesity prevalence among children and adolescents \\ from Northeast and Southeast regions of Brazil
}

\author{
Marcelo M. Abrantes ${ }^{1}$, Joel A. Lamounier ${ }^{2}$, Enrico A. Colosimo ${ }^{3}$
}

\begin{abstract}
Resumo
Objetivo: estudar a prevalência de sobrepeso e obesidade em crianças e adolescentes da região Sudeste e Nordeste.

Métodos: dados da pesquisa sobre padrões de vida, realizada pelo Instituto Brasileiro de Geografia e Estatística (IBGE) em 1997. Estudadas 3.317 crianças e 3.943 adolescentes agrupados em faixas etárias. Sobrepeso (adolescentes) e obesidade (crianças e adolescentes) foram definidos segundo recomendações da Organização Mundial de Saúde. Comparação de prevalências por faixa etária, sexo e região geográfica pelo teste $\mathrm{z}$, considerando 0,05 como nível de significância estatística.
\end{abstract}

Resultados: a prevalência de sobrepeso em adolescentes variou entre $1,7 \%$ no Nordeste, e $4,2 \%$ no Sudeste. A prevalência de obesidade em adolescentes variou entre $6,6 \%$ e $8,4 \%$, e em crianças entre $8,2 \%$ e $11,9 \%$, nas regiões Nordeste e Sudeste, respectivamente. Agrupando-se os dados das duas regiões, a prevalência no sexo feminino foi de $10,3 \%$ de obesidade entre crianças, $9,3 \%$ de obesidade e $3,0 \%$ de sobrepeso entre adolescentes. No sexo masculino, a prevalência foi de $9,2 \%, 7,3 \%$ e $2,6 \%$, respectivamente.

Conclusões: a prevalência de obesidade é menor no Nordeste, com significância estatística entre crianças e adolescentes com idade entre 2-17 anos. O mesmo ocorreu com a prevalência de sobrepeso entre os adolescentes. A diferença de prevalência de obesidade entre lactentes e adolescentes acima de 18 anos não foi estatisticamente significante. Entre lactentes, a obesidade é mais prevalente no sexo feminino. Nas outras faixas etárias, não houve diferença estatisticamente significante entre os sexos. A comparação dos resultados com os de outros estudos é difícil pela diversidade de critérios utilizados na classificação nutricional.

J Pediatr (Rio J) 2002; 78 (4): 335-40: obesidade, sobrepeso, infância, adolescência.

\begin{abstract}
Objective: to study the prevalence of obesity and overweight among children and adolescents from Northeast and Southeast regions of Brazil.
\end{abstract}

Methods: data was collected form Life Pattern Research conducted by the Brazilian Institute of Geography and Statistics (IBGE) in 1997. A sample of 3,317 children and 3,943 adolescents was studied according age groups. Overweight (adolescents) and obesity (adolescents and children) were defined according to World Health Organization recommendations. Z-test was used to compare prevalence among age groups, sex and geographic region. The cut-off point for statistical significance was 0.05 .

Results: overweight prevalence in adolescents was $1.7 \%$ in Northeast and $4.2 \%$ in Southeast. Obesity prevalence in adolescents was $6.6 \%$ and $8.4 \%$ in children and $8.2 \%$ and $11.9 \%$ in Northeast and Southeast, respectively. Considering both regions prevalence of obese female children was $10.3 \%$, the rate of obese adolescents was 9.3\% and overweight adolescents represented 3.0\%. Among males prevalence was $9.2 \%, 7.3 \%$ and $2.6 \%$, respectively.

Conclusions: obesity prevalence is lower in Northeast than Southeast among children with age from 2 to 17 years old. Overweight prevalence among adolescents was also lower in Northeast than in Southeast. The prevalence of obesity among younger than 2 and older than 18 years old was the same. Obesity prevalence is higher among breast-fed females. Among other age groups prevalence of obesity and overweight was the same for males and females. It is difficult to compare these results with other studies, since there are few populational studies and the criteria used to define overweight and obesity in children and adolescents varies in each study.

J Pediatr (Rio J) 2002; 78 (4): 335-40: obesity, overweight, childhood, adolescence.

\section{Introdução}

A obesidade é considerada, em países desenvolvidos, um importante problema de saúde pública, e pela OMS, uma epidemia global ${ }^{1}$. O aumento de sua prevalência em 
países em desenvolvimento, especialmente na América Latina, também já foi estudada, e em países como Índia e China o aumento de $1 \%$ na prevalência de obesidade gera 20 milhões de novos $\operatorname{casos}^{2}$. Está associada com hipertensão arterial, doença cardíaca, oesteoartrite, diabetes tipo 2 e alguns tipos de câncer, e seu impacto é mais pronunciado na morbidade do que na mortalidade ${ }^{1-3}$. Pessoas obesas, particularmente crianças e adolescentes, freqüentemente apresentam baixa auto-estima, afetando a performance escolar e os relacionamentos.

A prevalência de obesidade também está crescendo intensamente, na infância e na adolescência, e tende a persistir na vida adulta: cerca de $50 \%$ de crianças obesas aos seis meses de idade, e $80 \%$ das crianças obesas aos cinco anos de idade, permanecerão obesas ${ }^{4,5}$. Além disso, evidências científicas têm revelado que a aterosclerose e a hipertensão arterial são processos patológicos iniciados na infância, e nesta faixa etária são formados os hábitos alimentares e de atividade física ${ }^{6-8}$. Por isso, a preocupação sobre prevenção, diagnóstico e tratamento da obesidade tem-se voltado para a infância.

A antropometria é considerada o método mais útil para rastrear obesidade, por ser barato, não invasivo, universalmente aplicável, e com boa aceitação pela população ${ }^{1,9}$. Índices antropométricos são obtidos a partir da combinação de duas ou mais informações antropométricas básicas (peso, sexo, idade, altura) ${ }^{1}$. O uso do IMC para identificar adultos com sobrepeso e obesidade é consensual, e seu uso na avaliação nutricional de crianças e adolescentes começou a ser mais difundido após a publicação de Must et al. ${ }^{10,11}$, que apresentaram valores de percentis por idade e sexo. Esses valores são considerados atualmente como referência pela OMS para identificar sobrepeso e obesidade somente em adolescentes, não sendo recomendados para crianças, as quais devem ser avaliadas com o índice peso/altura ${ }^{1}$.

Dados brasileiros com relação à obesidade infantil são ainda escassos, e muitos autores estudam faixas etárias específicas (crianças ou adolescentes isoladamente), e muitas vezes com amostras não representativas da população ${ }^{12}$. No presente estudo, foi avaliada a prevalência de sobrepeso e obesidade em crianças e adolescentes das regiões Sudeste e Nordeste do Brasil.

\section{Metodologia}

Para o presente estudo, foram utilizados dados da pesquisa sobre padrões de vida (PPV) coletados em 1997, pelo Instituto Brasileiro de Geografia e Estatística (IBGE), em convênio com o Banco Mundial. Esta pesquisa avaliou as condições de moradia, tendências demográficas (migração, fecundidade e história de nascimentos), acesso aos serviços de educação e saúde, nutrição, condições de vida da população brasileira, além de dados de peso e altura ${ }^{13}$. A amostra foi constituída por 19.409 adultos e crianças resi- dentes em 5.000 domicílios, distribuídos em 554 setores censitários em municípios das regiões Nordeste e Sudeste do país. O processo amostral se deu em dois estágios de seleção, com estratificação das unidades primárias - setor da base geográfica do censo demográfico 1991 - e seleção proporcional a uma medida de tamanho, e seleção aleatória das unidades de segundo estágio domicílio. As medições antropométricas foram realizadas utilizando balanças de precisão dinamarquesas, marca Seca, modelo 890 , calibradas até $150 \mathrm{~kg}$, com intervalos de $1 \mathrm{~g}$. As medições da altura de todas as pessoas pesquisadas foram realizadas com antropômetros de madeira, com intervalos de $1 \mathrm{~mm}$, procedendo-se as medições de acordo com treinamento adotado na Pesquisa Nacional de Saúde e Nutrição ${ }^{13}$. Os dados obtidos foram disponibilizados em CD-ROM, pelo IBGE.

As informações referentes a peso, altura e sexo foram extraídos do banco de dados do IBGE e armazenados em um banco de dados secundário no software Epi-Info, versão $6.04^{14}$.

Dentre os 4.313 adolescentes estudados pelo IBGE, 370 $(8,6 \%)$ não tinham informações sobre peso e/ou altura. O mesmo ocorreu com $440(11,7 \%)$ crianças, das 3.757 iniciais. Sendo assim, a amostra final deste estudo foi de 7.260 indivíduos, sendo 3.317 crianças, e 3.943 adolescentes.

A definição de obesidade no presente estudo seguiu as recomendações da $\mathrm{OMS}^{1}$. As crianças foram consideradas obesas quando o escore $\mathrm{z}$ do índice peso/altura, calculado pelo software Epi-Info, foi maior que dois. Para os adolescentes, utilizou-se o IMC, calculado pela fórmula: peso/ (altura) $)^{2}$, segundo os valores propostos por Must et al. ${ }^{11,12}$. Considerado como sobrepeso o IMC igual ou acima do percentil 85 e menor que o percentil 95, e obeso o IMC igual ou acima do percentil 95. Para este fim, foi desenvolvido um programa no software Epi-Info que agrupava os adolescentes por sexo e idade.

A variável idade, calculada em meses, foi obtida pela diferença entre a data do exame antropométrico e a data de nascimento. Em seguida, as crianças e adolescentes foram agrupados em faixas etárias de uso corrente na literatura médica: lactente ( 0 a 1 anos), pré-escolar ( 2 a 6 anos), escolar (7 a 9 anos), adolescência precoce (10 a 14 anos), adolescência média (15 a 17 anos) e adolescência final (18 a 19 anos) ${ }^{15}$.

Usando o teste $\mathrm{z}$, equivalente do qui-quadrado, ${ }^{16} \mathrm{a}$ prevalência total de obesidade (sexo masculino e feminino juntos) da região Nordeste foi comparada com a da região Sudeste, em cada faixa etária, para verificar se a diferença era estatisticamente significante. Comparou-se também a prevalência total de obesidade (região Nordeste e Sudeste juntas) para verificar se existe diferença estatisticamente significante entre a prevalência do sexo masculino e a prevalência do sexo feminino. Foi considerado como nível de significância estatística o valor de $\mathrm{p}<0,05^{16}$. 


\section{Resultados}

A distribuição das crianças e adolescentes referente ao sexo foi de $50,4 \%$ masculino e $49,6 \%$ feminino. A média de idade foi de 122,9 meses (desvio-padrão de 68 meses), e a mediana de 126 meses. A amostra de crianças e adolescentes em cada idade (ano a ano), sexo e região geográfica foi praticamente a mesma.

A prevalência de crianças e adolescentes com sobrepeso e obesidade foi menor na região Nordeste, e a prevalência de obesidade foi maior em crianças (Tabela1).

Tabela 1 - Prevalência de sobrepeso e obesidade em crianças e adolescentes das regiões Nordeste e Sudeste do Brasil, 1997

\begin{tabular}{lcccc}
\hline & \multicolumn{2}{c}{ Sobrepeso } & \multicolumn{2}{c}{ Obesidade } \\
& Sudeste & Nordeste & Sudeste & Nordeste \\
\hline Crianças* & - & - & $11,9 \%$ & $8,2 \%$ \\
Adolescentes & $10,4 \%$ & $6,6 \%$ & $1,7 \%$ & $4,2 \%$ \\
\hline
\end{tabular}

* A OMS não apresenta definição de sobrepeso para crianças.

Analisando-se os dados das duas regiões conjuntamente, observa-se que prevalência de sobrepeso e obesidade foi maior no sexo feminino (Tabela 2).

Tabela 2 - Prevalência de sobrepeso e obesidade em crianças e adolescentes, agrupados por sexo, das regiões Nordeste e Sudeste do Brasil, 1997

\begin{tabular}{lcccc}
\hline & \multicolumn{2}{c}{ Sobrepeso } & \multicolumn{2}{c}{ Obesidade } \\
& Feminino & Masculino & Feminino & Masculino \\
\hline Crianças* & - & - & $10,3 \%$ & $9,2 \%$ \\
Adolescentes & $9,3 \%$ & $7,3 \%$ & $3,0 \%$ & $2,6 \%$ \\
\hline
\end{tabular}

* A OMS não apresenta definição de sobrepeso para crianças.

A prevalência de obesidade foi maior na região Sudeste quando comparada à prevalência da região Nordeste, com diferença estatisticamente significante, nas faixas etárias de pré-escolar $(\mathrm{p}<0,01)$, escolar $(\mathrm{p}<0,01)$, adolescência precoce $(\mathrm{p}<0,01)$ e média $(\mathrm{p}<0,05)$. A prevalência de sobrepeso também foi maior na região Sudeste, com diferença estatisticamente significante entre os adolescentes $(p<0,05)$ (Tabela 3).

Comparando-se a prevalência de obesidade no sexo masculino e feminino, no Brasil (regiões Nordeste e Sudeste juntas), encontramos maior prevalência no sexo femini-
Tabela 3 - Prevalência (em porcentagem) de sobrepeso e obesidade em crianças e adolescentes, agrupados por região geográfica, das regiões Nordeste e Sudeste do Brasil, 1997

\begin{tabular}{lcccc}
\hline & \multicolumn{2}{c}{ Sobrepeso } & \multicolumn{2}{c}{ Obesidade } \\
& Nordeste & Sudeste & Nordeste & Sudeste \\
\hline Lactente $^{\dagger}$ & - & - & 13,4 & 15,5 \\
Pré-escolar $^{\dagger}$ & - & - & 7,8 & $10,1^{*}$ \\
Escolar $^{\dagger}$ & - & - & 5,6 & $12,4^{*}$ \\
Precoce $_{\text {Média }}$ & 6,9 & $12,2^{*}$ & 2,4 & $7,2^{*}$ \\
Tardia & 6,3 & $9,3^{*}$ & 1,3 & $2,8^{*}$ \\
& 6,8 & $9,4^{*}$ & 0,8 & 1,5 \\
\hline
\end{tabular}

${ }^{*} p<0,05$ †A OMS não apresenta definição de sobrepeso para crianças.

no, com diferença estatisticamente significante somente entre os lactentes $(\mathrm{p}<0,01)$. Entre os escolares, o valor de $\mathrm{p}$ foi 0,07 . A diferença de prevalência de obesidade e sobrepeso entre os adolescentes do sexo masculino e feminino não apresentou significância estatística (Tabela $4 \mathrm{e}$ Figura 1).

\section{Discussão}

As recomendações da OMS de se usar o índice peso/ altura na avaliação nutricional de crianças não encontra aceitação unânime, sendo que, desde a publicação de Must et al. ${ }^{10,11}$, diversos autores apresentaram novas curvas de IMC, que já estão sendo usadas na avaliação nutricional de

Tabela 4 - Prevalência (em porcentagem) de sobrepeso e obesidade por sexo em crianças e adolescentes, agrupados por faixa etária, das regiões Nordeste e Sudeste do Brasil, 1997

\begin{tabular}{lcccc}
\hline & \multicolumn{2}{c}{$\begin{array}{c}\text { Sobrepeso } \\
\text { Feminino }\end{array}$} & \multicolumn{2}{c}{ Obesidade } \\
& Masculino & Feminino & Masculino \\
\hline Lactente $\ddagger$ & - & - & 19,0 & $10,2^{*}$ \\
Pré-escolar $\ddagger$ & - & - & 8,9 & 8,7 \\
Escolar ${ }^{\ddagger}$ & - & - & 7,3 & $9,3^{\dagger}$ \\
Precoce & 10,4 & 8,0 & 4,6 & 4,3 \\
Média & 7,9 & 7,4 & 2,4 & 1,5 \\
Tardia & 10,6 & $5,7^{*}$ & 1,2 & 1,1 \\
\hline
\end{tabular}

${ }^{*} p<0,05 \quad+p=0,07$

‡ A OMS não apresenta definição de sobrepeso para crianças. 


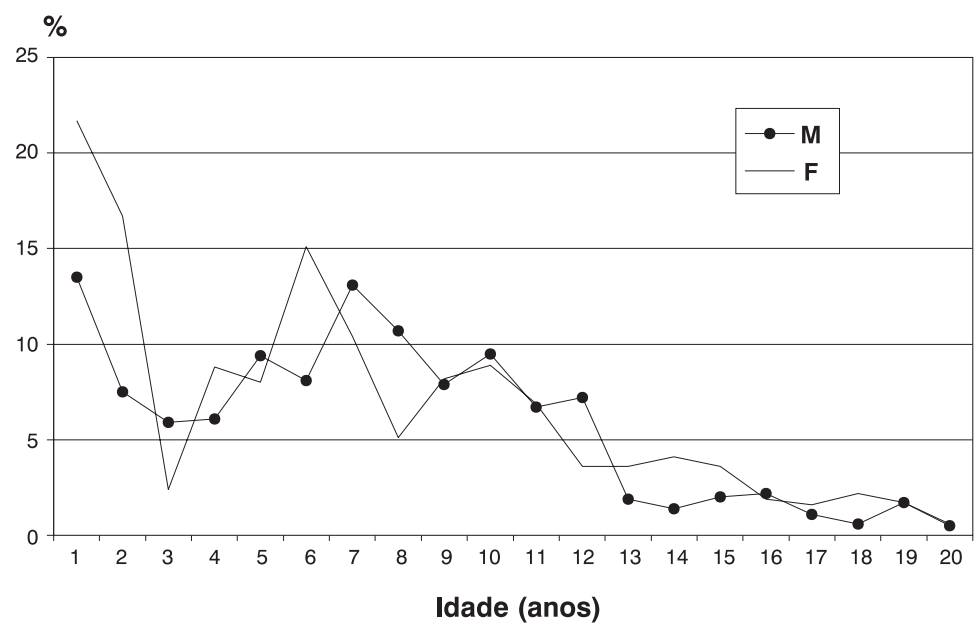

Figura 1 - Prevalência de obesidade por sexo e idade

crianças e adolescentes ${ }^{17}$. Devido a essa diversidade de critérios usados (valores de IMC diferentes), alguns autores consideram que uma estimativa internacional da prevalência e da tendência secular de obesidade pediátrica, bem como a comparação de estudos de prevalência, não sejam possíveis ${ }^{18}$. Outro estudo mostrou que resultados de estudos de prevalência de sobrepeso e obesidade baseados nos valores propostos por Cole et al. e Must et al. podem ser comparados ${ }^{19}$.

Dados brasileiros com relação à obesidade infantil são ainda limitados, e a ausência de unanimidade na definição de obesidade nesta faixa etária acarreta dificuldades na comparação das prevalências relatadas nos diversos estu$\operatorname{dos}^{12,20}$. Outro fato que limita a comparação é o de que a maior parte dos estudos nacionais são baseados em amostras de estudantes ou de ambulatórios médicos, portanto não representativas da população. Além disso, poucos analisaram conjuntamente crianças e adolescentes. Como a amostra do presente estudo é representativa da população das regiões Nordeste e Sudeste, e possui um tamanho amostral adequado, podemos extrapolar esses resultados para a população de crianças e adolescentes destas duas regiões geográficas do Brasil, e comparar com outros estudos nacionais.

A prevalência de obesidade na infância no presente estudo foi maior do que a descrita em outros estudos brasileiros. Na cidade de São Paulo, foi encontrada a prevalência de $3,8 \%$ de obesidade em 1.280 crianças entre zero e cinco anos de idade ${ }^{21}$. Em Pelotas, a prevalência de obesidade em 1.564 crianças com 12 meses de idade foi três vezes menor $(6,7 \%)^{22}$. A prevalência na infância $(0-10$ anos) descrita em uma favela de Maceió $(1,6 \%)$, foi cinco vezes menor ${ }^{23}$. A prevalência de obesidade em adolescentes do presente estudo foi semelhante à prevalência descrita em Belo Horizonte $(2,1 \%)^{23}$, e menor que a prevalência de outros estudos brasileiros, que variou entre $5,2 \%$ e $11,2 \%{ }^{23-25}$.

A prevalência de sobrepeso entre adolescentes encontrada neste estudo foi semelhante a encontrada em outros estudos: em Pelotas $(11,2 \%)^{22}$, Belo Horizonte $(5,7-6,3 \%)^{24}$ e Rio de Janeiro $(9,3-10,9 \%)^{25}$. Um estudo conduzido em Curitiba mostrou prevalência menor $(4,4 \%)^{26}$.

Com relação ao sexo, alguns autores descrevem maior prevalência de sobrepeso e obesidade no sexo feminino, entre os adolescentes ${ }^{26}$, mas o oposto também é descrito ${ }^{12}$. No presente estudo, a obesidade foi mais prevalente no sexo feminino somente entre lactentes, enquanto que a prevalência de sobrepeso foi maior entre as adolescentes do sexo feminino.

A comparação com estudos internacionais também é dificultada pelo agrupamento de dados em diferentes faixas etárias e pelo uso de diferentes curvas de IMC. A prevalência no presente estudo foi menor que a descrita em estudos australianos e americanos ${ }^{28,29}$.

Alguns autores descrevem que este início ocorre por volta dos 5-6 anos de idade, especialmente em meninas ${ }^{29}$. $\mathrm{Na}$ Arábia Saudita, a maior prevalência de sobrepeso e obesidade foi descrita entre os seis e dez anos de idade ${ }^{30}$. $\mathrm{Na}$ China, a maior prevalência de sobrepeso e obesidade entre adolescentes é relatada entre onze e doze anos ${ }^{30}$. No presente estudo, a maior prevalência de obesidade foi encontrada em lactentes, com outro pico entre os préescolares e declínio progressivo desta idade em diante (Figura 1).

No presente estudo, a prevalência de obesidade na infância foi sempre maior que a prevalência na adolescência (Figura 1). Esta diferença poderia ser explicada pelo uso 
de índices antropométricos diferentes: peso/altura na infância, e IMC na adolescência.

Outra explicação pode ser as diferentes fases do ritmo de crescimento normal das crianças e dos adolescentes. A maior prevalência de obesidade em lactentes pode ser explicada pelo fato de ocorrer nesta fase a repleção, que ocorre principalmente entre zero e nove meses de idade, e um ritmo de crescimento mais lento. A repleção é caracterizada por um aumento proporcionalmente maior do peso do que da altura, com aumento do subcutâneo, refletindo um armazenamento de gordura. A repleção ocorre também no início da adolescência, e pode levar a queixas de genitália hipodesenvolvida pelo acúmulo de gordura na região imediatamente inferior ao púbis ${ }^{31}$.

É descrito também que os pré-escolares apresentam duas fases distintas de crescimento: estirão, entre os dois e cinco anos, e crescimento lento, entre os cinco e sete anos de idade (observando o Figura, identificamos que a prevalência de obesidade foi maior nesta segunda fase). Entre os escolares, até o início da adolescência, a prevalência de obesidade manteve-se praticamente estável. O comportamento da prevalência de obesidade condiz com a idade de início de estirão: 10 anos nas meninas, e 12 anos nos meninos ${ }^{15}$.

Conclui-se que a prevalência de sobrepeso em adolescentes é maior no sexo feminino. A prevalência de obesidade é menor na região Nordeste entre crianças e adolescentes com idade entre 2-17 anos de idade. Na faixa etária de crianças abaixo de dois anos, e adolescentes acima de 18 anos, a diferença na prevalência da região Nordeste e Sudeste não foi estatisticamente significante. A comparação dos resultados deste estudo com os de outros é dificultada pela escassez de estudos populacionais nacionais, e pela diversidade de critérios utilizados.

\section{Referências bibliográficas}

1. World Health Organization. Physical status: the use and interpretation of anthropometry. Geneva: WHO; 1995.

2. Vischer TL, Seidell JC. The public health impact of obesity. Annu Rev Public Health 2001;22:355-75.

3. Eckersley RM. Losing the battle of the bulge: causes and consequences of increasing obesity. Med J Aust 2001;174:590-2.

4. Troiano RP, Flegal KM, Kukzmarski RJ, Campbell SM, Johnson CL. Overweight prevalence and trends for children and adolescents - The National and Nutrition Examination Surveys, 1963 to 1991. Arch Pediatr Adolesc Med 1995;149:1085-91.

5. Gortmaker SL, Dietz WH, Sobol AM, Wehler CA. Increasing pediatric obesity in the United States. Am J Dis Child 1987;141:535-40.

6. Mc Namara JJ, Malot MA, Stremple JF, Cutting RT. Coronary artery disease in combat casualties in Vietnam. JAMA 1971;216:1185-7.

7. Francischi RPP, Pereira LO, Freitas CS, Klopfer MM, Santos RC, Vieira P, et al. Obesidade: atualização sobre sua etiologia, morbidade e tratamento. Revista Nutrição 2000;13:17-28.
8. Story M, Evans M, Fabsitz RR, Clay TE, Rock BH, Broussard B. The epidemic of obesity in American Indian communities and the need for childhood obesity-prevention programs. Am J Clin Nutr 1999;69 Supl 4:747-54.

9. Goulart EMA, Corrêa EJ, Leão E. Avaliação do crescimento. In: Leão E, Corrêa E, Viana MB, Mota JAC, editores. Pediatria Ambulatorial. $3^{a}$ ed. Belo Horizonte: Coopmed; 1998. p. 71-94.

10. Must A, Dallal GE, Dietz WH. Reference data for obesity: 85 th and 95th percentiles of body mass index $\left(\mathrm{wt} / \mathrm{ht}^{2}\right)$ and triceps skinfold thickness. Am J Clin Nutr 1991;53:839-46.

11. Must A, Dallal GE, Dietz WH. Reference data for obesity: 85 th and 95th percentiles of body mass index $\left(\mathrm{wt} / \mathrm{ht}^{2}\right)$ and triceps skinfold thickness - a correction. Am J Clin Nutr 1991;54:773.

12. Balaban G, Silva GAP. Prevalência de sobrepeso e obesidade em crianças e adolescentes de uma escola da rede privada de Recife. J Pediatr (Rio J) 2001;77:96-100.

13. Ministério do Planejamento, Orçamento e Gestão. Pesquisa sobre Padrões de Vida 1996-1997. $2^{a}$ ed. Rio de Janeiro: IBGE; 1999.

14. Dean AG, Dean JA, Coloumbier D, Burton AH, Brendel KA, Smith DC. Epi Info, version 6.04: a word processing, database, and statistics program for public health on microcomputers. Centers for Disease Control and Prevention - World Health Organization; 1996.

15. Ferreira RA, Romanini MAV, Miranda SM, Beirão MMV. Adolescente: particularidades de seu atendimento. In: Leão E, Corrêa EJ, Viana MB, Mota JAC, editores. Pediatria Ambulatorial. $3^{\mathrm{a}}$ ed. Belo Horizonte: Coopmed; 1998. p. 49-56.

16. Soares JF, Siqueira AL. Introdução à Estatística Médica. $1^{\mathrm{a}}$ ed. Belo Horizonte: Departamento de Estatística - UFMG; 1999.

17. Abrantes MM, Lamounier JA, Colosimo EA. Índice de massa corporal para identificar obesidade na infância e adolescência: indicações e controvérsias. Revista Médica de Minas Gerais. No prelo.

18. Guillaume M. Defining obesity in childhood: current practice. Am J Clin Nutr 1999;7 Supl 1:126-30.

19. Abrantes MM, Lamounier JA, Colosimo EA. IMC na obesidade: recomendações da OMS X tendências atuais. Anais do III World Congress of Pediatric Nutrition; 2001 jul 6-9; São Paulo, SP. São Paulo; 2001. p. 29-30.

20. Monteiro CA, Conde WL. Tendência secular da desnutrição e da obesidade na infância na cidade de São Paulo (1974-1996). Rev Saude Publica 2000;34 Supl 6:8-12.

21. Post CL, Victora CG, Barros FC, Horta BL, Guimarães PRV. Desnutrição e obesidade infantis em duas coortes de base populacional no Sul do Brasil: tendências e diferenciais. Cad Saude Publica 1996;12 Supl 1:49-57.

22. Florêncio TMMT, Ferreira HS, França APT, Cavalcante JC, Sawaya AL. Obesity and undernutrition in a very-low-income population in the city of Maceió, northeastern Brazil. Br J Nutr 2001;86:277-83.

23. Ribeiro RQC, Oliveira RG, Colosimo EA, Bogutchi TF, Laomunier JA. Prevalência da obesidade em escolares adolescentes na cidade de Belo Horizonte. Resultados parciais do II Estudo Epidemiológico. Anais do Simpósio: Obesidade e anemia carencial na adolescência. Instituto Danone; 2000 jun 89; Salvador, BA. Salvador; 2000. p.237-38.

24. Castro IRR, Engstrom EM, Anjos LA, Azevedo AM, Silva CS. Perfil nutricional dos alunos da rede municipal de educação da cidade do Rio de Janeiro. Anais do Simpósio: Obesidade e anemia carencial na adolescência. Instituto Danone; 2000 jun 89; Salvador, BA. Salvador; 2000. p.231-32.

25. Von der Heyde MED, Amorim STSP, Lang RMF, Von der Heyde R. Perfil nutricional de adolescentes da cidade de Curitiba. Anais do Simpósio: Obesidade e anemia carencial na adolescência. Instituto Danone; 2000 jun 8-9; Salvador, BA. Salvador; 2000. p. 227. 
26. Mokhtar N, Elati J, Chabib R, Bour A, Elkaki K, Schlossman NP, et al. Diet culture and obesity in northern Africa. J Nutr 2001;131 Supl 3:887-92.

27. Al-Shammari SA, Khoja T, Gad A. Community-based study of obesity among children and adults in Ryadh, Saudi Arabia. Food and Nutrition Bulletin 2001;22:178-83.

28. Booth ML, Wake M, Armstrong T, Chey T, Hesketh K, Mathur S. The epidemiology of overweight and obesity among Australian children and adolescents, 1995-97. Aust N Z J Public Health 2001;25:162-9.

29. Hanley AJG, Harris SB, Gittelsohn J, Wolever TMS, Saksvig B, Zinman B. Overweight among children and adolescents in a Native Canadian community: prevalence and associated factors. Am J Clin Nutr 2000;71:693-700.
30. Chunning C. Fat intake and nutritional status of children in China. Am J Clin Nutr 2000;72 Supl 5:1368-72.

31. Longui CA. Crescimento normal. In: Monte O, Longui CA, Calliari LEP, editores. Endocrinologia para o pediatra. $2^{\mathrm{a}}$ ed. São Paulo: Atheneu; 1998. p. 3-10.

Endereço para correspondência:

Prof. Joel Alves Lamounier

Dep. de Pediatria - Faculdade de Medicina da UFMG

Av. Alfredo Balena, 190

CEP 30130-100 - Belo Horizonte, MG

Fone: (31) 9992.3193 / Fax: (31) 3282.6324 